\title{
Contrôle De L'inflation En Regime De Change Fixe: Le Cas De La Communaute Economique Et Monetaire d'Afrique Centrale (CEMAC)
}

\author{
Tangakou Soh Robert \\ Mba Fokwa Arsène \\ Akanga Reuben Johnson
}

Faculté des Sciences Economiques et de Gestion, Université de Dschang, Cameroun. Département d'Analyse et de Politique Economique

doi: 10.19044/esj.2017.v13n10p379 URL:http://dx.doi.org/10.19044/esj.2017.v13n10p379

\begin{abstract}
This paper focuses on the determinants of inflation under different policy rules and fixed exchange rate regime, the example of the CEMAC zone. The purpose of this paper is to check the behaviour of inflation in fixed exchange rate regime for a flexible targeting period and a period of strict targeting. The data used are mainly from the World Bank, in «the book of world development indicators» contained in the CD -ROM (WDI 2015). Working for the periods 1977-1994, 1995-2012 and 1977-2012, the analyses was done with a dynamic panel that has the distinction of being among the independent variables, the endogenous variable lagged one or more periods. The endogenous variable is the rate of inflation. Estimates made from the Arellano and Bond (1991) method, it is clear that during the period (19771994) of flexible inflation targeting, money supply, trade balance and the exchange rate are the main determinants of inflation. During the period (1995-2012) of strategy of strict inflation targeting, the main determinants of inflation are the benefits of natural resources, the trade balance and the economic crisis. The determinants of inflation have opposing effects of a match type to another and it is the combination of these effects for each variable that shows the different effects of the determinants of inflation over the period. The exchange rate increased the rate of inflation over the first sub-period (1977-1994) and throughout the entire period (1977-2012). In times (1995-2012) of strict inflation targeting, these negative effects were mitigated at the expense of economic growth. Countries with fixed exchange rate regime should not adopt a strict policy of inflation targeting, but should alternate with the growth objective by facilitating financing for investments.
\end{abstract}

Keywords: Inflation targeting, determinants of inflation, dynamic panel 


\section{Resume}

Cet article est centré sur les déterminants du taux d'inflation sous différentes règles de politique monétaire et en régime de change fixe, l'exemple pris est celui de la zone CEMAC. L’objectif du présent papier est de vérifier le comportement de l'inflation en régime de change fixe pendant une période de ciblage flexible et une période de ciblage strict. Les données utilisées proviennent principalement de la Banque Mondiale, dans «le livre des indicateurs mondiaux de développement» contenu dans le CD -ROM (WDI-2015). Travaillant pour les périodes 1977-1994, 1995-2012 et 19772012, l'analyse a été faite avec un panel dynamique qui a la particularité d'avoir parmi les variables indépendantes, la variable endogène retardée d'une ou de plusieurs périodes. La variable endogène est le taux d'inflation. Des estimations faites à partir de la méthode d'Arellano et Bond (1991), il ressort que pendant la période (1977-1994) de ciblage flexible de l’inflation, la masse monétaire, la balance commerciale et le taux de change sont les principaux déterminants de l'inflation. En stratégie de ciblage strict (19952012) de l'inflation, les principaux déterminants de l’inflation sont les bénéfices tirés des ressources naturelles, la balance commerciale et la crise économique. Les déterminants de l'inflation ont des effets qui s'opposent d'un type de ciblage à l'autre et c'est la conjugaison de ces effets pour chaque variable qui donne les différents effets des déterminants de l'inflation sur toute la période. Le taux de change fait augmenter le taux d'inflation sur la première sous-période (1977-1994) et sur la période toute entière (19772012). En période (1995-2012) de ciblage stricte de l’inflation, ces effets négatifs ont été atténués au détriment de la croissance économique.

Les pays à régime de change fixe ne devraient pas adopter une stratégie stricte de ciblage d'inflation, mais devraient alterner avec objectif de croissance par facilitation du financement pour les investissements.

Mots clés : Ciblage de l'inflation, déterminants de l'inflation, panel dynamique

\section{INTRODUCTION}

Depuis les années 90, La conduite de la politique monétaire a connu des immenses changements passant d'un système de contrôle direct des taux d'intérêt et des agrégats monétaires à un système de régulation de la monnaie au moyen des taux d'intérêt directeurs. Cette évolution s'inscrit dans le cadre de l'objectif de stabilité des prix assigné à la politique monétaire pour assurer la bonne conduite de cette politique.

La lutte contre l'inflation est devenue indispensable pour les banques centrales à l'échelle mondiale. Cela découle d'un accord qu'une politique 
fondée sur des règles de conduite est plus fiable qu'une politique discrétionnaire et dépendante (Alesina, 1989; Alesina et Summers, 19993).

Il importe de noter que la discrétion et la non-crédibilité de la politique monétaire engendrent le problème d'incohérence temporelle time inconsistency problem décrite par Kydland et Prescott (1977), R.Barro et D.Gordon (1983). La crédibilité de l'autorité monétaire est alors le corollaire de la cible d'inflation pour que les agents comprennent et font confiance à la stratégie de la banque centrale. Il en découle ainsi deux règles de politique monétaire à savoir les règles d'instruments ${ }^{70}$ et les règles d'objectif ${ }^{71}$. Mais on se demande quelle règle adopter dans le cas d'une économie ouverte avec un régime de change fixe? Dans le cas où on opte pour une règle d'objectif, la règle doit-elle être stricte ou flexible? Les réponses à ces questions qui se posent ont été obtenues dans le cas des pays à taux de change flexibles (Bail, 1998; Svensson, 1997; Clarida et al., 2002)

Selon l'idée de Fischer, S. (2001), les régimes de taux de change fixes ou ancrés à d'autres monnaies ont été un facteur de toutes les grandes crises financières qui ont frappé les marchés des changes des pays émergents ces dernières années comme le Mexique à la fin de 1994, la Thaïlande, l'Indonésie et la Corée de Sud en 1997, la Russie et le Brésil en 1998, l'Argentine et la Turquie en 2000 et de nouveau la Turquie en 2001.

La politique monétaire d'un pays ou d'une zone devrait servir au développement de ce pays ou de cette zone. Selon Shari (2007), la stabilité réelle et non la stabilité des prix est ultimement l'objectif le plus important pour attirer les investisseurs et réaliser le développement durable. Ainsi, Une attention exagérément ou exclusivement portée à la stabilité des prix peut avoir une incidence négative sur la croissance.

Selon Chauvin(2012), à l'exception de la République Centrafricaine, la croissance et l'équilibre externe de toutes les économies de la CEMAC qui ont été marqués par la prépondérance des exportations de biens primaires, notamment de pétrole, traduisent bien la faible diversification de l'économie. Or, l’épuisement des recettes pétrolières pourrait révéler des vulnérabilités importantes en cas de non-diversification de la structure des exportations.

\footnotetext{
${ }^{70}$ Ces règles qui peuvent être soit implicites, soit explicites, selon qu'elles sont définies avec ou sans les variables anticipées, font référence à la reconnaissance d'une forme fonctionnelle permettant de déterminer le niveau des instruments à un moment donné. Les principales règles d'instruments sont la règle de McCallum (1987) qui considère comme instrument l'agrégat monétaire de base et comme cible le PIB nominal. La règle de HendersonMcKibbin (1993) la règle de Taylor (1993) caractérisé par le fait que l'instrument est le taux d'intérêt à court terme et comme cible le taux d'inflation.

${ }^{71}$ Quant aux règles d'objectif, leur fondement de base réside dans le respect d'un objectif fixé par les autorités monétaires. Une règle d'objectif vise à minimiser, dans une fonction de perte, l'écart entre le niveau anticipé de la variable cible et le niveau objectif de ladite variable. L'objectif ici peut-être soit un objectif final, soit un objectif intermédiaire.
} 
Lorsque l'inflation est ciblée en régime de change fixe, la politique monétaire devient «stérile» et la politique économique paralysée car les autorités monétaires ne peuvent plus prendre des décisions de politique monétaire afin d’influencer le développement de l'activité réelle. L’objectif de ce papier est de vérifier le comportement de l'inflation en régime de change fixe pendant une période de ciblage flexible et une période de ciblage strict. Le reste de la rédaction sera dans l'ordre des sections suivantes: littérature, méthodologie, résultats et enfin conclusion.

\section{REVUE DE LA LITTÉRATURE}

\section{II.1. REVUE DE LA LITTÉRATURE THEORIQUE}

Selon Carré (2011), le ciblage de l'inflation nait en 1989 en NouvelleZélande. Plus de vingt ans après, ce régime de politique monétaire domine la littérature, a été adopté par plus de 25 pays et constitue la norme du Fonds Monétaire International. Pour la littérature standard, il est une créature de la « science » théorique, l'incohérence temporelle en particulier. L’histoire démontre qu'il s'inscrit aussi dans le cadre plus large des réformes gouvernementales s'inspirant plutôt de l'Ecole d'Harvard ${ }^{72}$. Plus encore, l'histoire révèle que ces théories jouent un rôle limité dans la création du ciblage de l'inflation apparaissant comme un " art » pragmatique quasiment sans fondation théorique. Cette nature d'innovation au regard de la théorie est une originalité dans l'histoire des régimes monétaires récents. Les théories à la base du ciblage d'inflation et les déterminants de l'inflation quant à eux datent de très longtemps.

Bodin (1568) rejette l'idée selon laquelle la hausse des prix serait seulement due à la dépréciation de l'unité de compte. Pour obtenir la même quantité de biens qu'au début du siècle, il faut livrer une quantité supérieure de métal. Non seulement cet auteur explique la hausse des prix par l'afflux d'un métal que reçoit d'abord l'Espagne, mais, il montre comment cette hausse se répercute en France. Le supplément de métal dont dispose l'Espagne lui permet d'importer des produits français, d'autant plus que le renchérissement des produits nationaux stimule les importations, et d'employer une importante main-d'œuvre française attirée par des salaires élevés. Le paiement des importations et le rapatriement des salaires des émigrés procurent un apport de métal à la France où les prix augmentent à leur tour.

Hume (1970), effectue une présentation mécanique des effets d'une variation du stock de monnaie sur les prix qui nous rapproche des formulations modernes de la théorie quantitative. Selon cet auteur, si en une

${ }^{72}$ L'apparition du ciblage d'inflation s'inscrit en Nouvelle-Zélande dans le cadre de la réforme de l’Etat lancée par le gouvernement travailliste en 1984. 
nuit les quatre cinquièmes de la monnaie circulant en Grande-Bretagne disparaissent, les prix devraient baisser dans les mêmes proportions. Au contraire, si par miracle cette quantité de monnaie quintuplait dans la nuit, l'effet inverse se produirait.

Pour James (1970), l'inflation est un phénomène global et non pas strictement monétaire, global par ses causes, car étant un excès de la demande globale. Global pour ses conséquences, en ce sens qu'elle affecte l'économie dans son ensemble, modifie les prix, les structures des revenus et l'orientation de la production.

Les travaux sur le coefficient pass-through ${ }^{73}$ (Goldberg et Knetter, 1996), distinguent généralement trois canaux par lesquels les variations du taux de change affectent les prix domestiques. Dans le premier canal, les variations des taux de change affectent les prix locaux directement à travers les biens dans le panier du consommateur. Dans le deuxième canal, la transmission se fait à travers les prix des biens intermédiaires utilisés comme moyen de production. A travers ce dernier, le taux de change affecte le coût de production des entreprises. Alors, pour un niveau donné de concurrence, en supposant que le bien vendu est un bien dont la demande est assez rigide aux variations de prix, ces producteurs peuvent répercuter l'augmentation du prix des intrants sur les consommateurs. Dans le dernier canal, le taux de change affecte les prix des biens domestiques libellés en devise.

Selon Mantsie (2003), les causes de l'inflation sont multiples et, dans le cas de la $\mathrm{CEMAC}^{74}$, toutes n’ont pas de raison d'être. L'analyse économique nous enseigne qu'il existe trois sources d'inflation. La première résulte d'un excès de la demande sur l'offre ${ }^{75}$, la seconde d'un renchérissement des coûts des facteurs de production ${ }^{76}$ et la troisième est la résultante des structures socio- économiques ${ }^{77}$.

\section{II.2. REVUE DE LA LITTÉRATURE EMPIRIQUE}

Couharde et al. (2011), en travaillant sur «Taux de change d’équilibre et processus d’ajustement du franc CFA» ont utilisé comme méthode la

\footnotetext{
${ }^{73}$ Le pass-through est le phénomène par lequel la dépréciation d'une monnaie affecte les prix dans une économie.

${ }^{74}$ Communauté Economique et Monétaire d’Afrique Centrale.

${ }^{75}$ L'inflation par la demande s'identifie à un état de l'économie dans lequel le réajustement de l'équilibre, par suite d'une perturbation due à un accroissement des dépenses (par suite d’une injection supplémentaire de liquidité par exemple) se fait par les prix stimulés à la hausse.

${ }^{76}$ l'augmentation des prix des facteurs de production se répercute sur le prix de revient total des biens qui par une spirale stimule les prix à la hausse.

${ }^{77}$ la rigidité des structures économiques expliquerait l'élévation des prix dans la mesure où l'offre demeure inélastique .face à la demande.
} 
Cointégration en panel $\left(\mathrm{DOLS}^{78}\right)$, MCE à transition lisse en panel sur la période 1985-2007. Ils ont eu pour principale conclusion une Hétérogénéité des mésalignements entre les pays, asymétrie dans l'effet des mésalignements.

Charalambos et Tsangarides (2006), en tavaillant sur «le régime du taux de change réel pour le franc CFA dans les zones CEMAC et $\mathrm{UEMOA}^{79}$ " pour la période 1970 à 2005 ont utilisé un modèle $\mathrm{VAR}^{80}$ avec cointégration de Johansen, ils ont eu comme principal résultat l'absence de mésalignements en 2005, un retour à l'équilibre 2 fois plus lent pour la CEMAC.

Gary et al. (1995), ont analysé les facteurs majeurs influençant l'inflation au Nigéria en employant la cointégration et un modèle à correction d'erreur et des données portant sur la période de 1960 à 1993. Ils ont utilisé le revenu réel, la masse monétaire, la pluviométrie annuelle et le taux de change bilatéral Naira/dollar US. Ils ont trouvé que la croissance de la masse monétaire, induite par les politiques budgétaires expansionnistes, explique à un fort pourcentage le processus inflationniste au Nigéria. Les autres facteurs sont la dévaluation du naira et les conditions agroclimatiques.

Mosayed et Mohammad (2009) ont examiné les déterminants de l'inflation en Iran pour des données portant sur la période de 1971 à 2006. En utilisant un model Autorégressif à retard échelonné, ils ont conclu que le taux d'intérêt, le taux de change, le produit national brut la variation entre prix domestique et prix étranger, une variable qui capte les effets de la guerre d'Iran ou d'Irak sont les déterminants majeurs de l'inflation en Iran. Toutes ces variables contribuent positivement à l'augmentation des prix domestiques en Iran.

Abidemi et Malik (2010) ont analysé les relations simultanées entre l'inflation et ses principaux déterminants au Nigéria pour la période de 1970 à 2007. La méthodologie utilisée a été la cointégration de Johansen suive du modèle à correction d'erreur. Leur étude révèle que le produit intérieur brut, l'offre de monnaie, les importations, l'inflation retardée d'une période et le taux d'intérêt sont positivement reliés à l'inflation.

Furrukh et al (2011), ont recherché les déterminants de l'inflation au Pakistan, pour atteindre cet objectif, l'étude a porté sur les séries temporelles pour la période allant de 1972 à 2010. L'approche par la cointégration de Johansen et le modèle à correction d'erreur ont été utilisés pour les estimations. Les relations de causalité ont été observées à partir du test de causalité de Granger. Les données sur les variables macroéconomiques ont

\footnotetext{
${ }^{78}$ Double Moindre Carré

${ }^{79}$ Union Economique et Monétaire Ouest-Africaine.

${ }^{80}$ Vecteur Auto-Regessif.
} 
été obtenues à partir des publications statistiques du Pakistan. Les résultats révèlent qu'à long terme, l'indice de prix à la consummation est positivement influencé par l’offre de monnaie, le produit intérieur brut, les importations et les dépenses gouvernementales. Par contre les recettes gouvernementales réduisent le niveau de prix au Pakistan dans l'emsemble.

Sahadudheen (2012), en travaillant sur les déterminants de l'inflation en Inde, a utilisé une de cointégration suivie de modèle vectoriel à correction d'erreur. Après avoir trouvé un ajustement de long terme des variables, il resort de son etude que le produit intérieur brut et l'offre de monnaie ont un effet positif sur l'inflation à long terme, alors que le taux d'intérêt et le taux de change ont un effet négatif.

Selon Gavrel (1991), lors du passage aux changes flexibles, l'Etat recouvre le contrôle de la création monétaire et peut ainsi desserrer sa contrainte de budget intertemporelle. Mais en raison de la spéculation qui provoque un «saut» des encaisses monétaires à la date de rupture et d'un effet type «courbe de Laffer», le recours au seigneuriage est limité ; il existe un taux de croissance «optimal» de la masse monétaire (au sens de solvabilité de l'Etat) au-delà duquel toute augmentation tend à dégrader la solvabilité de l'Etat. Ainsi deux conséquences s’en déduisent :d’une part, allonger arbitrairement la phase de fixité du change en soutenant la dette publique par la perspective toujours repoussée d'une monétisation massive est exclu, d'autre part lorsque l'inflation mondiale est élevée (donc le taux de croissance initial de l'offre de monnaie domestique), alors même que les finances publiques sont dégradées, le passage aux changes flexibles s'accompagnera non d'une accélération mais d'une décélération de l'inflation domestique et donc d'une appréciation du change.

\section{METHODOLOGIE}

Les données utilisées dans cet article sont toutes de source secondaire, provenant de la publication annuelle de la Banque Mondiale, dans «le livre des indicateurs mondiaux de développement» contenu dans le CD -ROM (WDI-2015); de la BEAC (Rapports d'Activité de la BEAC); de l'INS. L'étude couvre la période allant de 1977 à 2012. Cette période est divisée en deux sous-périodes. La première sous-période est la période de ciblage flexible ${ }^{81}$ de l'inflation. Elle part de 1977 à 1994 et est marquée par une expansion, une crise et un ensemble de réformes qui se sont succédées.

\footnotetext{
${ }^{81}$ Ici Il est essentiel de distinguer la fonction de perte sociale, c'est-à-dire la fonction qui vise un ou plusieurs objectifs, de celle à un seul objectif cible. Ainsi, à l'instar de K. Rogoff (1985), on peut attribuer une fonction objective à la banque centrale, l'autorité qui formule et met en oeuvre la politique monétaire. La fonction ainsi attribuée est normalement choisie de façon à minimiser la fonction de perte sociale.
} 
La deuxième sous-période est la période de ciblage strict ${ }^{82}$ de l'inflation. Elle va de 1995 à 2012 et est caractérisée par une reprise, une mise en œuvre des réformes et des difficultés pour un réel décollage. Ce choix se justifie par le souci d'intégrer les diverses évolutions qu'a connues la politique monétaire au sein de la CEMAC. Compte tenu des grands changements (utilisation exclusive des instruments indirectes de politique monétaire, dévaluation du taux de change, entrée en vigueur de la stratégie de ciblage d'inflation) dans la politique monétaire des Etats de la zone CEMAC en 1994, il est très important de faire les analyses sur deux sous-périodes et sur la période entière.

\section{III.1 PRESENTATION DU MODELE}

Le modèle utilisé dans cet article est un modèle de régression à une équation indépendante, qui comporte plusieurs variables exogènes. Il sera présenté tour à tour la variable endogène et les variables exogènes.

-Variable endogène : Le taux d'inflation (TINFL) : Pour mesurer l'inflation, nous utilisons l'indice du prix à la consommation à l'instar de Claus (1997), lorsqu'il recherche la relation entre l'inflation et la croissance ; Blix (1995) lorsqu'il recherche la relation entre l'inflation observée et la croissance de la masse monétaire; Engone (2003), lorsqu'il recherche le niveau cible d'inflation dans la zone CEMAC. L'inflation renseigne sur la stabilité des prix. Elle a une influence indéterminée à l'avance sur l'activité économique car tout dépend de sa source et des anticipations des agents économiques.

\section{-Variables exogènes :}

- Le taux de change (TCH): Pour mesurer le taux de change d’équilibre, nous avons utilisé le taux de change réel comme Dupuy (2013) lorsqu'il met en œuvre des mesures de «quantitave easing» (QE) ou détente quantitative afin d'améliorer les conditions de financement dans l'ensemble de l'économie. il mesure le niveau de compétitivité internationale du pays et peut par conséquent être déterminant, surtout pour les firmes exportatrices. En régime de change fixe et pour une mesure de dévaluation réussie, le taux de change doit baisser l'inflation.

- le taux de croissance (TPIBR): Pour nous renseigner sur la croissance, nous utilisons le PIB réel par tête comme Fischer (1993),

\footnotetext{
${ }^{82}$ La forme de ciblage strict est mise en exergue quand la banque centrale est appelée à réaliser la cible avec précision, indépendamment des implications pour d'autres objectifs. Etant donné que le critère central retenu par les autorités monétaires est clairement un objectif d'inflation, la banque centrale s'efforce de minimiser une fonction dans laquelle l'inflation est la variable cible (fonction de perte de la banque centrale)
} 
Sarel (1996) et Mantsie (2003) lorsqu’ils déterminent un seuil à partir duquel l'inflation devient nocive pour l'économie.

- La balance courante (BC) : Pour mesurer la balance courante, nous utilisons le solde de la balance commerciale à l'instar de Duasa (2007) lorsqu'il recherche les déterminants de la balance commerciale malaysienne. elle est mesurée par la balance commerciale dans ce travail. Selon que la balance commerciale soit excédentaire ou déficitaire, elle fait augmenter ou baisser l'inflation.

- L'emploi (EMPL): l'emploi a été mesuré avec le niveau de l'emploi comme l'a fait Pichette (1998) lorsqu’elle détermine si les réactions asymétriques sont observables au niveau du marché du travail au Canada. L’emploi est mesuré par le ratio emploi-population qui est la proportion de la population d'un pays qui a un emploi. L'emploi peut faire baisser ou augmenter l'inflation selon que coût du travail est bas ou élevé.

- les crédits à l'économie (CE) : ils sont mesurés par le crédit intérieur net qui est la somme des crédits nets accordés au secteur public non financier et au secteur privé ainsi que d'autres comptes. Cette définition est des Statistiques financières internationales et autres fichiers de données du FMI. Lorsqu'on est dans le circuit de production, les crédits font baisser l'inflation.

- Le taux d'intérêt (TI): Le taux d'intérêt réel est le taux d'intérêt débiteur ajusté en fonction de l'inflation telle que mesurée par le déflateur du PIB. Cette définition est des Statistiques financières internationales et autres fichiers de données du FMI. Selon que le taux d'intérêt est fort ou faible, il freine ou booste l'inflation.

- La masse monétaire $\left(\mathbf{M}_{2}\right)$ : La monnaie et quasi-monnaie désignent la somme des devises à l'extérieur des banques, des dépôts à vue autres que ceux du gouvernement central, et les dépôts à terme fixe, d'épargne et en devises étrangères des secteurs résidents autres que le gouvernement central. Cette définition de la masse monétaire est souvent qualifiée de $\mathrm{M}_{2}$; elle correspond à celle des Statistiques financières internationales (SFI) du Fonds monétaire international (FMI). La masse monétaire entre dans le circuit des échanges et stimule l'inflation.

- Les investissements (INV): Les investissements privés financent les dépenses brutes du secteur privé (notamment les agences privées à but non lucratif) avec les ajouts à ses avoirs intérieurs fixes. Cette définition est celle des données sur les comptes nationaux de la Banque mondiale et fichiers de données sur les comptes nationaux de 
l'OCDE $^{83}$. Par l'augmentation de l'offre des biens et service et la création de la valeur, les investissements font baisser l'inflation.

- Les bénéfices tirés des ressources naturelles (OIL): Le total des bénéfices tirés des ressources naturelles correspond à la somme des bénéfices tirés du pétrole, du gaz naturel, du charbon (anthracite et houille), des minéraux et des forêts. Cette définition provient de «La richesse changeante des nations : mesurer le développement durable dans le nouveau millénaire», publié par la Banque mondiale en 2011. Les bénéfices tirés des ressources naturelles s’ils ne sont pas en parie réinvestis sont source d'inflation.

- La crise (CRIEC): la crise est le ralentissement de l'activité économique, comme Paulo (2001), Cordemans et al. (2012), elle est mesurée par une variable «dummy» marquée par 1 pour les années de crise et par 0 pour les années d'absence de crise.

- La dévaluation (DEV): la dévaluation intègre ici la décision qui a fait changer le taux de change en le fixant à 100 Fcfa pour $1 \mathrm{~F}$ français en 1994. Elle est mesurée par une variable «dummy» marquée par 1 pour les années à partir de la dévaluation et par 0 pour les années d'avant dévaluation. En l'absence d'une réelle production et des débouchés automatiques, la dévaluation ne peut être que nocive pour une économie.

\section{III.2. SPECIFICATION DU MODELE}

L'équation de l'inflation retenue dans le présent travail de recherche prend sa base chez Phillips (1958), nous-nous inspirons des travaux de Nubukpo (2002).

L'indice des prix à la consommation (IPC), écrit sous forme loglinéaire, est fonction du coût des biens domestiques (IPD) et celui des biens importés (IPM) exprimé en franc CFA.

$\operatorname{logIPC}=\alpha \log$ IPD $+(1-\alpha) \operatorname{logIPM}$

avec $0<\alpha<1$

Le prix domestique dépend des tensions existant sur le marché de la monnaie et celui des biens et services. Par conséquent, il sera fonction d'une part, de l'offre de monnaie $\left(\mathrm{M}^{\mathrm{s}}\right)$ et de la demande $\left(\mathrm{M}^{\mathrm{d}}\right)$ et, d'autre part, du gap de production.

$\log I P D=\Omega_{1}\left(\log M^{s}-\log M^{d}\right)+\Omega_{2}(\log P I B R-\log P I B \circ)$

avec $\Omega_{1}, \Omega_{2}>0$

L'offre de monnaie dépend à son tour, des taux d'intérêt directeurs de la BEAC et du PIB réel, le taux d'intérêt directeur étant constitué du taux du marché monétaire (IM) et du taux de prise en pension (IPS).

\footnotetext{
${ }^{83}$ Organisation de Coopération et de Développement Economique.
} 
$\mathrm{M}^{\mathrm{s}}=G(\mathrm{IM}, \mathrm{IPS}, \mathrm{PIBR})$

Ou $\mathrm{M}^{\mathrm{s}}=-\beta_{1}$ IM $-\beta_{2}$ IPS $+\beta_{3} \log$ PIBR

avec $\beta_{1}, \beta_{2}, \beta_{3}>0$

La demande de monnaie dépend du revenu réel des agents économiques,

$\mathrm{M}^{\mathrm{d}}=\mathrm{F}(\mathrm{PIBR})$

Ou $\mathrm{M}^{\mathrm{d}}=\beta_{4} \log$ PIBR $\quad$ avec $\beta_{4}>0$

En substituant les équations (4) et (6) dans (2), puis (2) dans (1), il vient que

$\log$ IPC $=\alpha \Omega_{1}\left(-\beta_{1}\right.$ IM- $\beta_{2}$ IPS $+\beta_{3} \log$ PIBR $-\beta_{4} \log$ PIBR $)+\alpha \Omega_{2}(\log$ PIBR -

$\left.\log \mathrm{IIBR}^{\circ}\right)+(1-\alpha) \log I \mathrm{PM}$

En supposant un modèle linéaire pour l'équation (7) et en remplaçant le taux d'intérêt directeur par le taux d'intérêt réel (TI) pour que l'inflation soit celle qui explique l'activité économique réelle, l'évolution du taux d'inflation suivra la fonction suivante :

IPC $=\mathrm{H}$ (TI, PIBR, PIBR ${ }^{\circ}$, IPM)

Dans l'équation (8), la variable PIBR $^{\circ}$ qui est censée déterminer le PIB potentiel sera remplacée par l'ensemble des variables qui sont susceptibles d'expliquer le PIB potentiel à savoir : l'offre de crédits (CE), la masse monétaire $\left(\mathrm{M}_{2}\right)$, les investissements (INV), le total des bénéfices tirés des ressources naturelles, la valeur ajoutée de l'agriculture, la balance courante (BC) et l'emploi (EMPL). L'indice du prix des importations (IPM) a été remplacé par le taux de change pour tenir compte de l'influence et de la compétitivité des échanges sur l'inflation.

Le PIB réel est susceptible de traduire un effet demande au sein de l'équation. Le signe attendu de cette variable est non déterminé, dans la mesure où la valeur de son paramètre dépend des évolutions relatives de l'offre de monnaie, de la demande de monnaie et du choc d'offre. La part importée de l'inflation est également une variable explicative potentielle dans la mesure où une hausse des prix des produits importés se répercute sur les prix domestiques, du fait notamment d'un comportement de marge de la part des importateurs. L'indice des prix des produits importés, est remplacé par le taux de change (TCH) FCFA / dollar.

Finalement, l'équation d'inflation se mettra sous la forme :

IPC =H (TI, PIBR, $\mathrm{M}_{2}$, INV, OIL, BC, EMPL, TCH, CE)

En tenant compte de la forte dynamique des variables monétaires ou des variables en relation avec des variables monétaires, le taux d'inflation sera expliqué par sa variable retardée IPC(r) où le retard $r$ permet d'instrumenter le taux d'inflation. Les effets de la crise et de la dévaluation sur l'atteinte des objectifs de politiques économiques ont été pris en compte par des variables «dummies». Par ailleurs, pour tenir compte des dimensions spatiale et temporelle de notre modèle, nous prenons respectivement les 
indices i et t. Ce qui nous conduit à la spécification ci-après, en prenant pour proxy de l'IPC le taux d'inflation noté TINFL et pour proxy de PIBR la croissance économique notée TPIBR, on a :

$$
\begin{aligned}
\text { TINFL }_{i t}=\alpha_{0} & +\alpha_{1} \text { TINFL }_{i t-r}+\alpha_{2} T I_{i t}+\alpha_{3} \text { TPIBR } \\
& +\alpha_{5} \text { INV }_{i t}+\alpha_{4} \text { M }_{2 i t} \\
& +\alpha_{10} C E_{i t}+\alpha_{11} \text { CRIEC }_{i t}+\alpha_{7} B C_{i t}+\alpha_{8} E E V_{i t} \\
& +\varepsilon_{1 i t}
\end{aligned}
$$

Avec $\alpha_{0}$ la constante, $\alpha_{1}$ à $\alpha_{12}$ les coefficients des variables, $\varepsilon_{1 i t}$ le terme d'erreur. $\boldsymbol{\varepsilon}_{\mathbf{i t}}=\mathbf{U}_{\mathbf{i}}+\mathbf{V}_{\mathbf{t}}+\mathbf{W}_{\mathbf{i t}}$ où $\mathbf{U}_{\mathbf{i}}$ désigne un terme constant au cours

\begin{tabular}{|c|c|c|}
\hline \multirow[b]{3}{*}{$\begin{array}{l}\text { Variables } \\
\text { explicatives }\end{array}$} & \multirow[b]{3}{*}{ Mesures des variables } & \multirow{2}{*}{$\begin{array}{l}\text { Variables expliquées } \\
\text { TINFL }\end{array}$} \\
\hline & & \\
\hline & & Signes attendus \\
\hline $\mathrm{TCH}$ & Taux de change réel & - \\
\hline TPIBR & PIB par habitant & $+/-$ \\
\hline $\mathrm{BC}$ & Balance extérieure des biens et services & $+/-$ \\
\hline EMPL & $\begin{array}{l}\text { Ratio emploi-population, personnes âgées } \\
\text { de } 15 \text { ans et plus }\end{array}$ & $+/-$ \\
\hline $\mathrm{CE}$ & Crédit intérieur brut & - \\
\hline TI & Taux d’intérêt réel & $+/-$ \\
\hline $\mathrm{M}_{2}$ & Monnaie et quasi-monnaie & $+/-$ \\
\hline INV & Formation brute du capital fixe & - \\
\hline OIL & $\begin{array}{l}\text { Total des bénéfices tirés des ressources } \\
\text { naturelles }\end{array}$ & $+/-$ \\
\hline CRIEC & Variable dummy & + \\
\hline $\mathrm{DEV}$ & Variable dummy & + \\
\hline
\end{tabular}
de la période ne dépendant que de l'individu $\mathbf{i}, \mathbf{V}_{\mathbf{t}}$ un terme ne dépendant que de la période $\mathbf{t}, \mathbf{W}_{\mathrm{it}}$ un terme aléatoire croisé.

Tableau 1 : Récapitulatif des signes attendus

\section{PRESENTATION DES RESULTATS}

\section{IV.1. Test de stationnarité}

Le test d'Imp Pesaran et Shin (IPS) (2003) est utilisé pour déceler la présence éventuelle de racines unitaires. Ce test qui repose sur la moyenne des statistiques de Dckey-Fuller Augmenté est effectué avec constante, puis avec constante et trend. De ce fait, l'hypothèse alternative pour le test sur les variables est la stationnarité avec une constante non-nulle puis la stationnarité avec une constante non nulle et présence de trend.

Du tableau du test de stationnarité ci-dessous, li ressort que toutes les séries retenues sont stationnaires à niveau avec constante mais pas avec trend car toutes les probabilités attachées à nos séries sont inférieures au seuil de signification de $1 \%$. Nous passons ainsi aux estimations afin d'apprécier les différents effets entre nos variables. 
Tableau 2 : test de racine unitaire des séries

\begin{tabular}{|c|c|c|c|c|c|c|c|c|c|c|c|c|c|}
\hline SERIES & \multicolumn{7}{|c|}{ Tests de stationnarité d'IIPS effectués à niveau avec constante } & \multicolumn{6}{|c|}{ Tests de stationnarité d'IPS effectués à niveau avec constante et trend } \\
\hline & \multicolumn{2}{|c|}{ 1ère sous-période } & \multicolumn{2}{|c|}{ 2ème sous-période } & \multicolumn{3}{|c|}{ Période entière } & \multicolumn{2}{|c|}{ 1ère sous-période } & \multicolumn{2}{|c|}{ 2ème sous-période } & \multicolumn{2}{|c|}{ Période entière } \\
\hline & \multicolumn{2}{|c|}{ IPS w-stat=31,5849 } & \multicolumn{2}{|c|}{ IPSw-stat=29,4300 } & \multicolumn{3}{|c|}{ IPSw-stat=45,1530 } & \multicolumn{2}{|c|}{ IPS w-stat=32,3703 } & \multicolumn{2}{|c|}{ IPS w-stat=29,8717 } & \multicolumn{2}{|c|}{ IPS w-stat=47,2222 } \\
\hline & \multicolumn{2}{|c|}{ MoyenneADF=-7,6639 } & \multicolumn{2}{|c|}{ Moyenne ADF=-9,9928 } & \multicolumn{3}{|c|}{ Moyenne ADF=-11,889 } & \multicolumn{2}{|c|}{ Moyenne ADF=-8,6232 } & \multicolumn{2}{|c|}{ Moyenne ADF=-9,1249 } & \multicolumn{2}{|c|}{ Moyenne ADF=-12,314 } \\
\hline & \multicolumn{2}{|c|}{ IPSw-prob=0,0000 } & \multicolumn{2}{|c|}{ IPSw-prob=0,0000 } & \multicolumn{3}{|c|}{ IPSw-prob=0,0000 } & \multicolumn{2}{|c|}{ IPSw-prob=0,0000 } & \multicolumn{2}{|c|}{ IPSw-prob=0,0000 } & \multicolumn{2}{|c|}{ IPSw-prob=0,0000 } \\
\hline & t-stat & prob & t-stat & prob & t-stat & prob & & t-stat & prob & t-stat & prob & t-stat & prob \\
\hline TINFL & $-5,2247$ & tis & -9,6948 & & \begin{tabular}{|c|}
$-10,874$ \\
\end{tabular} & & 0 & $-9,0387$ & 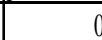 & $-8,4508$ & 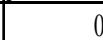 & $-11,009$ & 0 \\
\hline TPIBR & $-7,7725$ & ; & $-5,6514$ & & $-11,76$ & & 0 & $-5,0672$ & 0,0003 & $-8,8239$ & 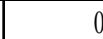 & $-11,753$ & 0 \\
\hline EMPL & $-2,0666$ & 0,0086 & $-8,967$ & & $-8,9959$ & & 0 & $-7,832$ & 0,0086 & $-4,0227$ & 0,0107 & $-8,9605$ & \\
\hline $\mathrm{BC}$ & $-9,2779$ & c & $-11,494$ & & $-14,863$ & & 0 & $-10,622$ & 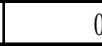 & $-10,499$ & 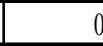 & $-14,829$ & 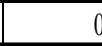 \\
\hline $\mathrm{TI}$ & $-2,4931$ & 0,001 & $-10,238$ & & $-4,1435$ & & 0 & $-9,352$ & 0,001 & $-2,8568$ & 0,181 & $-4,1663$ & 0,0059 \\
\hline M2 & $-8,5494$ & 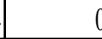 & $-7,7958$ & & $-10,012$ & & 0 & $-6,4134$ & 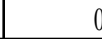 & $-11,172$ & 0 & $-10,063$ & \\
\hline $\mathrm{CE}$ & $-8,0312$ & 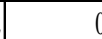 & $-13,33$ & & $-16,999$ & & 0 & $-12,063$ & 0 & $-11,818$ & 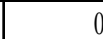 & $-16,972$ & 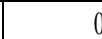 \\
\hline OIL & $-8,334$ & c & $-8,8126$ & & $-12,257$ & & 0 & $-7,6799$ & 0 & $-9,969$ & 0 & $-12,237$ & \\
\hline TCH & $-14,848$ & 0,0001 & $-11,769$ & & $-13,518$ & & 0 & $-7,3803$ & 0,0001 & $-8,4963$ & 0 & $-13,49$ & \\
\hline INV & $-6,9045$ & i & 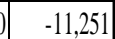 & & $-13,31$ & & 0 & $-9,761$ & 0 & $-9,8359$ & 0 & $-13,448$ & 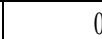 \\
\hline
\end{tabular}

\section{IV.2. Estimations}

La méthode d'estimation retenue ici est celle d'Arellano et Bond (1991). Les modèles dynamiques se caractérisent par la présence d'une ou de plusieurs valeurs retardées de la variable endogène parmi les variables explicatives. Dans ces modèles, la présence de la variable dépendante retardée ne permet pas d'utiliser les techniques économétriques standards. L'estimation des modèles dynamiques par les méthodes classiques (MCO et Within) donne des estimateurs biaisés et non convergents à cause de la corrélation entre la variable endogène retardée et le terme d'erreur. Pour contourner cette difficulté, plusieurs propositions ont été faites, la plus populaire étant celle fondée sur la méthode des moments généralisées développée par Arellano et Bond (1991). Sa popularité s'explique par plusieurs avantages et notamment la prise en compte d'effets fixes inobservables, de l'endogénéité des variables explicatives, ainsi que la possibilité de travailler avec des panels non cylindrés. Le tableau cidessous nous donne le résultat de nos estimations.

Lorsqu'il existe une plus forte corrélation entre variable monétaire et activité économique qu'entre inflation et activité économique, l’objectif visé doit être l'inflation, il est nécessaire que cet objectif soit intermédiaire. En annexe, lorsque chaque fois que pour les trois découpages périodiques on compare la corrélation entre inflation et croissance avec les corrélations tour à tour entre croissance et taux d'intérêt, croissance et masse monétaire puis croissance et crédits; on comprend bien que la zone CEMAC a eu des raisons de passer de la stratégie de ciblage flexible de l'inflation a une stratégie de ciblage strict. Mais l'échec de cette politique est dû à la non prise en compte de certains prérequis à savoir le régime de change flexible, la souveraineté 
monétaire et l'amélioration des échanges commerciaux qui passe par la transformation des matières premières et la diversification des partenaires commerciaux.

De toutes nos variables, on constate que le taux d’intérêt, les crédits, les investissements, l'emploi n'ont pas des coefficients significatifs à $10 \%$. Le taux d'inflation retardé d'une période a un coefficient significatif à 1\% pour la première sous-période et la période entière, ce coefficient est positif, donc le taux d'inflation passé s’il est élevé a tendance à faire grimper le taux d'inflation futur. Ceci se comprend bien lorsque les biens et services produits le sont à partir des facteurs de production dont les coûts sont très élevés.

Le taux d'intérêt réel a pendant la deuxième sous-période, un effet négatif et significatif à un seuil de $10 \%$ sur l'inflation, on attendait un signe neutre. Lorsque le taux d'intérêt augmente, l'inflation diminue, ça devrait être le cas seulement à court terme, mais si ça l'est à long terme, c'est parce qu'en zone CEMAC le taux d'intérêt est plus utilisé dans une optique de cible d'inflation à moins de $3 \%$ que dans une optique d'encouragement des investissements.

La masse monétaire a un coefficient négatif et significatif au seuil de $10 \%$ pendant la première sous-période, dans ce cas où le signe attendu est indéterminé, une croissance de la masse monétaire entraine une diminution de l'inflation. Ceci est d'autant plus vrai qu'une partie de la masse monétaire qui augmente passe toujours dans les circuits de production. Ainsi l'offre de biens et service qui augmente réduit l'inflation liée à la demande. Donc en période de ciblage flexible, la masse monétaire a permis de réduire l’inflation.

Les bénéfices tirés de la production des ressources naturelles ont un effet négatif (le signe neutre était attendus) et significatif au seuil de 1\% sur l'inflation pendant la deuxième sous-période. Une baisse des bénéfices tirés des ressources naturelles fait grimper le taux d'inflation. Ce qui traduit la baisse des prix des matières première et le désavantage compétitif que les pays de la zone CEMAC ont à exporter les matières premières et à importer les produits manufacturés. Donc en période de ciblage strict, les bénéfices tirés des ressources naturelles ont plutôt dégradé la stabilité des prix.

Sur la période toute entière, à un seuil de signification de $5 \%$, une augmentation de $100 \%$ du taux de croissance fait croître le taux d'inflation de $7,814 \%$, donc on peut dire que le taux de croissance n'est pas une véritable source d'inflation en zone CEMAC.

Le taux de change a un coefficient positif (signe inattendu) et significatif à un seuil de $1 \%$ sur la première sous-période et sur la période entière, une augmentation du taux de change sur la période fait augmenter l'inflation. En effet, l'objectif selon lequel le taux de change qui s’appréciait après une dévaluation ou une dépréciation de la monnaie devait rendre 
l'économie plus compétitive n’a toujours pas profité aux économies de la zone CEMAC à cause de leur incapacité à produire des biens manufacturés à forte valeur ajoutée et de qualité. Les effets négatifs de l'appréciation du taux de change qui a occasionné la dévaluation en 1994 se sont étendus sur toute la période d'étude. En période de ciblage stricte de l'inflation, ces effets négatifs ont été atténués au détriment de la croissance économique.

La question sur les mérites des régimes de change fixe ou flexible sur la capacité à promouvoir la croissance ou à stabiliser l'économie date de plusieurs années bien avant l'effondrement du système de Bretton Wood. Les premiers travaux sur les zones monétaires optimales qui ont vu le jour au début des années 60 (Mundell, 1961 ; McKinnon, 1963; Kenen, 1969) ont alors mis en évidence l'importance de la prise en considération des spécificités et des caractéristiques structurelles dans un pays donné ainsi que le degré d'asymétrie des chocs entre partenaires commerciaux. Le modèle traditionnel de Mundell-Fleming a ensuite souligné la dichotomie entre chocs nominaux et réels comme principal déterminant du choix du régime de change. Encore plus récemment, plusieurs auteurs comme Aghion et al (2004) ont insisté sur les implications de l'intégration financière et de la hausse considérable dans les mouvements de capitaux et sur le fait qu'elles sont un élément clé dans la détermination du choix du régime de change pour des économies de plus en plus insérées dans l'environnement financier international.

En outre, notons que l'incohérence temporelle comme source d'inefficacité et comme principal déterminant du biais inflationniste, et a mis en exergue le rôle de la crédibilité dans la détermination du choix du régime de change (Sfia, 2007). La fixité du taux de change est de ce fait considérée comme substitut de la crédibilité des autorités monétaires insuffisamment crédibles. Le choix d'un régime de change est donc perçu comme un arbitrage entre crédibilité des autorités monétaires par la fixité du taux de change et absorption des chocs par les fluctuations du taux de change.

Le choix d'un régime de change est alors déterminé par : la Théorie de la zone monétaire optimale; la nature des chocs dans l'économie; la trinité impossible ( la trinité impossible ou encore de trinité économique contraint les autorités monétaires entre trois objectifs à savoir : fixer le taux de change pour stabiliser les prix, libre mobilité des capitaux et jouir d'une autonomie dans la conduite de la politique monétaire dans l'objectif d'assurer la stabilité macroéconomique) ${ }^{84}$; le péché originel (désigne l'incapacité pour un pays en général et pour les économies émergentes en particulier à emprunter à l'extérieur dans leurs propres monnaies. Ce qui les

${ }^{84}$ D’après Sfia (2007), à cause d'une globalisation accrue des marchés de capitaux, l'hypothèse de la trinité impossible implique aujourd'hui la disparition future des régimes du milieu, et donc par une dynamique vers les solutions en coin. 
conduit à accumuler des dettes en devises avec d’importants retombés sur les politiques macroéconomiques et la stabilité financière); l'effet de Passthrough (l'effet pass-through est défini comme le degré par lequel les fluctuations dans les taux de change sont transmises au niveau général des prix dans le pays et représentent un élément important dans le choix du régime de change pour les économies émergentes comme pour les économies les plus avancées).

La balance courante est négativement (on attendait un signe neutre) corrélée à au taux d'inflation dans la première sous-période et positivent corrélé au taux d’inflation dans la deuxième sous-période. Ceci s’explique tout simplement par le fait que la balance courante n’est pas déficitaire à la première sous-période mais est déficitaire à la deuxième sous-période. Les pays de la zone CEMAC importent aujourd'hui plus qu'ils n'exportent, en le faisant ils importent aussi l'inflation extérieure. La dévaluation est positivement corrélée au taux d’inflation sur toute la période. La dévaluation n'ayant pas permis aux économies de la zone CEMAC d'être plus compétitive, a favorisé la hausse des prix. Donc lorsqu'on applique une stratégie de ciblage stricte de l'inflation, la balance courante a un effet inflationniste.

La crise économique a un coefficient positif (signe attendu) et significatif au seuil de $1 \%$ et à $5 \%$ respectivement pendant la deuxième souspériode et la période entière, la crise a donc contribué à l'augmentation du taux d'inflation sur notre période d'étude. Les entreprises existantes n'ont pas beaucoup subi le coup de la crise à cause du transfert des capitaux qui se dirigent aujourd'hui vers les pays qui ont des technologies innovantes d'une part, d'autre part à cause de la faiblesse du tissu productif. En période de ciblage strict, la crise est plus inflationniste qu'en période de ciblage flexible.

Ce sont les consommateurs des biens et services qui ont toujours payé le prix de la crise car la production insuffisante des biens et services en interne, les revenus qui baissent et même la préférence et la dépendance pour les biens manufacturés importés ont contribué à amplifier l’inflation. La crise influence l'inflation en occasionnant la rareté qui rend la vie et les facteurs de production très chers. Ce modèle est globalement significatif à un seuil de 1\% donc les résultats sont valides dans l'ensemble. 
Tableau 5.3 : Estimation de l'équation de l'inflation

\begin{tabular}{|c|c|c|c|}
\hline \multirow[t]{3}{*}{ VARIABLES EXOGENES } & \multicolumn{3}{|c|}{ VARIABLE DEPENDENTE } \\
\hline & $\begin{array}{l}1^{\text {ère }} \text { sous- } \\
\text { période }\end{array}$ & $\begin{array}{l}2^{\text {ème }} \text { sous- } \\
\text { période }\end{array}$ & $\begin{array}{l}\text { Période } \\
\text { entière }\end{array}$ \\
\hline & TINFL & TINFL & TINFL \\
\hline TI & $\begin{array}{l}-0,0002822 \\
(-0,53) \\
{[0,596]}\end{array}$ & $\begin{array}{l}-0,00168^{*} \\
(-1,65) \\
{[0,099]}\end{array}$ & $\begin{array}{l}-0,0003757 \\
(-0,86) \\
{[0,387]}\end{array}$ \\
\hline TPIBR & $\begin{array}{l}0,1200084 \\
(2,40) \\
{[0,017]}\end{array}$ & $\begin{array}{l}0,00093 \\
(0,03) \\
{[0,973]}\end{array}$ & $\begin{array}{l}0,0673329 * * \\
(2,28) \\
{[0,023]}\end{array}$ \\
\hline $\mathrm{M}_{2}$ & $\begin{array}{l}-1,423988^{*} \\
(-1,34) \\
{[0,182]}\end{array}$ & $\begin{array}{l}0,08533 \\
0,17) \\
{[0,862]}\end{array}$ & $\begin{array}{l}-0,2670715 \\
(-0,49) \\
{[0,623]}\end{array}$ \\
\hline INV & $\begin{array}{l}0,0256632 \\
(1,34) \\
{[0,216]}\end{array}$ & $\begin{array}{l}0,00843 \\
(0,78) \\
{[0,439]}\end{array}$ & $\begin{array}{l}0,0148161 \\
(1,17) \\
{[0,244]}\end{array}$ \\
\hline OIL & $\begin{array}{l}0,0021347 \\
(0,11) \\
{[0,914]}\end{array}$ & $\begin{array}{l}-0,02964 * * * \\
(-2,98) \\
{[0,003]}\end{array}$ & $\begin{array}{l}-0,0153922 \\
(-1,34) \\
{[0,179]}\end{array}$ \\
\hline $\mathrm{BC}$ & $\begin{array}{l}-0,000631 * \\
(-1,89) \\
{[0,056]}\end{array}$ & $\begin{array}{l}0,00073^{*} \\
(1,81) \\
{[0,071]}\end{array}$ & $\begin{array}{l}-0,0002716 \\
(-1,01) \\
{[0,315]}\end{array}$ \\
\hline EMPL & $\begin{array}{l}-2,23148 \\
(-0,82) \\
{[0,410]}\end{array}$ & $\begin{array}{l}-0,73256 \\
(-0,81) \\
{[0,419]}\end{array}$ & $\begin{array}{l}0,2434835 \\
(0,25) \\
{[0,799]}\end{array}$ \\
\hline TCH & $\begin{array}{l}0,3729904^{* * *} \\
(10,24) \\
{[0,000]}\end{array}$ & $\begin{array}{l}0,02332 \\
(0,51) \\
{[0,611]} \\
\end{array}$ & $\begin{array}{l}0,2945912 * * * \\
(11,04) \\
{[0,000]}\end{array}$ \\
\hline $\mathrm{CE}$ & $\begin{array}{l}-7,584951 \\
(-0,88) \\
{[0,377]}\end{array}$ & $\begin{array}{l}-1,13951 \\
(-0,88) \\
{[0,377]}\end{array}$ & $\begin{array}{l}-0,0768838 \\
(-0,30) \\
{[0,761]}\end{array}$ \\
\hline CRIEC & $\begin{array}{l}0,485448 \\
(0,32) \\
{[0,749]}\end{array}$ & $\begin{array}{l}3,12288^{* * *} \\
(3,03) \\
{[0,002]}\end{array}$ & $\begin{array}{l}2,04315^{* *} \\
(2,04) \\
{[0,041]}\end{array}$ \\
\hline DEV & 1 & 1 & $\begin{array}{l}2,131423^{* *} \\
(2,20) \\
{[0,028]}\end{array}$ \\
\hline TINFL (-1) & $\begin{array}{l}0,3180867 * * * \\
(3,09) \\
{[0,002]}\end{array}$ & $\begin{array}{l}-0,11910 \\
(-0,15) \\
{[0,882]}\end{array}$ & $\begin{array}{l}0,321642 * * * \\
(5,90) \\
{[0,000]}\end{array}$ \\
\hline Statistiques critiques & $\begin{array}{l}2,57 \text { pour } 1 \% \\
1,96 \text { pour } 5 \% \\
2,57 \text { pour } 10 \% \\
\end{array}$ & $\begin{array}{l}2,57 \text { pour } 1 \% \\
1,96 \text { pour } 5 \% \\
2,57 \text { pour } 10 \%\end{array}$ & $\begin{array}{l}2,57 \text { pour } 1 \% \\
1,96 \text { pour } 5 \% \\
2,57 \text { pour } 10 \%\end{array}$ \\
\hline Observations & 96 & 96 & 204 \\
\hline Test de Sargan (p-value) & 0,3142 & 0,5862 & 0,5348 \\
\hline $\begin{array}{l}\text { Test d'autocorrélation de second ordre (p- } \\
\text { value) }\end{array}$ & 0,049 & 0,459 & 0,109 \\
\hline
\end{tabular}


*, ** et *** sont les significativités respectivement à $10 \%, 5 \%$ et $1 \%$. Les nombres qui croisent les variables sont les coefficients assujettis à chaque variable indépendante. Dans les parenthèses nous avons les valeurs des statistiques «Z» calculées et dans les crochets, nous avons les valeurs des probabilités.

En comparant la significativité du coefficient pour les variables qui ont au moins un coefficient significatif et l'ampleur (grandeur en nombre) du coefficient pour les variables qui n'ont aucun coefficient significatif, on peut dire qu'en stratégie de ciblage stricte de l'inflation, la croissance, les investissements et le taux de change ont tendance à réduire l'inflation. Cependant, le taux d'intérêt, la masse monétaire, les bénéfices tirés des ressources naturelles, la balance commerciale, l'emploi, les crédits, la crise économique ont tendance à réduire l'inflation pendant la stratégie de ciblage stricte. On observe exactement le contraire pendant la période de ciblage flexible de l'inflation.

\section{CONCLUSION}

Enfin, il est intéressant de rappeler que la politique monétaire doit servir au développement. Le taux d'inflation et le taux de change sont les deux grands prix ${ }^{85}$ de la politique monétaire, dans un cadre de politique monétaire souveraine, les autorités monétaires devraient pouvoir agir sur au moins un de ces deux principaux prix afin d'influencer positivement l'activité économique. Pendant la période de ciblage flexible de l'inflation, la masse monétaire, la balance commerciale et le taux de change sont les principaux déterminants de l'inflation. En stratégie de ciblage strict de l'inflation, les principaux déterminants de l'inflation sont les bénéfices tirés des ressources naturelles, la balance commerciale et la crise économique. Les déterminants de l'inflation ont des effets qui s'opposent d'un type de ciblage à l'autre et c'est la conjugaison de ces effets pour chaque variable qui donne les différents effets des déterminants de l'inflation sur toute la période. Le taux de change fait augmenter le taux d'inflation sur la première souspériode et sur toute la période d'étude. Le manque de marge de manœuvre (le franc CFA étant évaluer à parité fixe à l'euro, l'inflation étant ciblée à long terme) ne peut qu'enfoncer ou maintenir l'économie à un état embryonnaire. Selon Jahati (2007), les pays à régime de change fixe ne devraient pas adopter une stratégie stricte de ciblage d'inflation, mais devraient alterner avec objectif de croissance par facilitation du financement pour les investissements. Dans ce cas l'objectif final de la politique monétaire doit être la quantification du volume de monnaie nécessaire dans l'économie.

${ }^{85}$ On devrait pouvoir agir sur l'un ou l'autre pour modifier l'activité économique. 
L'inflation et le taux de change doivent être des objectifs intermédiaires. S'il existe une plus forte corrélation entre monnaie et activité économique qu'entre inflation et activité économique, l'objectif intermédiaire visé doit être l'inflation. Dans le cas contraire, l'objectif intermédiaire visé doit être le taux de change. Si c'est le taux d'inflation qui est visé, on peut opter pour une stratégie de ciblage d'inflation à court ou à moyen terme selon les opportunités économiques présentes. Si le taux de change qui est visé comme objectif intermédiaires, il faut pouvoir choisir le régime de change comme suit : préférer un régime de change flexible dans le cas où la population et la demande locale des biens et services sont très importante ; opter pour un régime de change fixe ou intermédiaire au cas où la population et la demande locale des biens et services sont faibles.

\section{References:}

1. Abidemi, O. I. and Malik, S. A. A., «Analysis of Inflation and its determinant in Nigeria», Pakistan Journal of Social Sciences, (2010), 7(2), 97-100.

2. Aftalion F. et Losq E. : Les taux de change, Que Sais-je ? , PUF 1e édition, Paris. (1985).

3. Alesina, A. «Politics and business cycles in industrial democracies», Economic Policy, (1989), volume 4, issue, 8, PP. 57-87.

4. Alesina, A., Summers, L. «Central bank independence and macroeconomic performance», Journal of Money, Credit, and Banking, volume 25, issue 2, (1993), pp.157-162.

5. Arellano M., Bond F., « Some tests of specification for panel data: Monte Carlo evidence and an application to employment equations », Review of Economic Studies, vol. 58, n²2, (1991), pp. 277-297.

6. Baffes, J.; Elbadawi, I A. ; O'Connell, Stephen A., , «Singleequation estimation of the equilibrium real exchange rate», Policy Research Working Paper, Series 1800, The World Bank. (1997)

7. Bail, L., «Policy Rules for Open Economies» NBER- Business cycles series, volume 31, (1998), p: 127.

8. Barro, R. et Gordon, D., " Rules, Discretion and Reputation in a Model of Monetary Policy ", Journal of Monetary Economics ${ }^{\circ}$ 12, (1983).

9. Blix M., «Underlying Inflation: A Common Trend Approach», in Sveriges Rikbank Arbets rapport, $\mathrm{n}^{\circ} 23$ (1995).

10. Bodin, J., (1568), Une réponse aux paradoxes de M. de Malestroit touchant l'enchérissement de toutes choses.

11. Bouoiyour, J. et Kuikeu, O., «Pertinence de la dévaluation du Franc CFA de janvier 1994: Une évaluation par le taux de change réel 
d’équilibre. Cas de l'économie camerounaise», MPRA Paper, 31357, University Library of Munich. (2007).

12. Richard Claridaa, Jordi Gali, Mark GertIer «A simple framework for international monetary policy analysis» in Journal of Monetary Economies volume 49, (2002), pp : 879-904.

13. Carré, E. «une histoire du ciblage d'inflation» Document de travail du CEPN, (2011), Juillet

14. Charalambos, G. ; Tsangarides, C. G. ; Yasser, A., «FEER for the CFA Franc», IMF Working Papers, 06/236, International Monetary Fund. (2006).

15. Chauvin S. Macroéconomie et Développement, Division Analyse macroéconomique et risque pays, Département de la Recherche, AFD (2012), Novembre.

16. Claus I., «A Measure of Underlying Inflation in the United States», in Bank of Canada-Working Paper, (1997) Septembre.

17. Couharde C.; Coulibaly I.; Damette O., «Taux de change d’équilibre et processus d'ajustement du franc CFA», (2011), congres.afse.fr/docs/2011/967172ccicod_v11.pdf.

18. Cordemans, N. et Ide, S. « La politique monétaire aux États-Unis et dans la zone Euro durant la crise» BNB Revue économique, (2012), Juin.

19. Duasa, J. «Determinants of Malasian Trade Balance: An ARDL Bound Testing Approach», Journal of Economic Cooperation, 28; 3, pp21-40, (2007).

20. Dupuy, M. «les effets des politiques de quantitative Easing sur le taux de change :les enseignements de l'expérience américaine» LAREFI Working paper, (2013), Janvier.

21. Engone S., «la cible d’inflation en zone CEMAC», (2003).

22. Fischer S: " The Role of Macroeconomic Factors in Growth», Journal of Monetary. (1993).

23. Fischer, S., « Régimes de taux de change: le bipolarisme est-il justifié? », Finance et Développement, (2001), juin, p. 5.

24. Furrukh, B.; Shahbaz, N.; Kalsoom Y. «determinants of inflation in pakistan: an econometric analysis using johansen co-integration approach», Australian Journal of Business and Management Research, vol $1 \mathrm{~N}^{0} 5$, 2011, August, PP. 71-82.

25. Gary G. Moser, «The Main Determinants of Inflation in Nigeria». IMF Staff Papers, Vol. 42, No. 2 (1995), June.

26. Gavrel, F. « Changes fixes, changes flexibles et solvabilté de l’État» Annales d'économie et de statistique- $\mathrm{N}^{0}$ 24. (1991). 
27. Goldberg P.K.; Knetter M.M. «Goods Prices and Exchange Rates: What have we learned» NBER Working paper, (1996), pp. 58 62.

28. Hume, D.; Writings on Economics, (ed.), Rotwein, E., Madison, Wis.: University of Wisconsin Press, (1970).

29. James E., Problèmes monétaires d'aujourd'hui, Paris, $2^{\text {ème }}$ édition, (1970).

30. Kahn. M et M. Knight: Stabilization Programs in Developing Countries: A Formal Framework, (1991), in Kahn M., Montiel P., Haque N. eds «Macroeconomic Models for Adjustment in Developing Countries», IMF, Washington D.C., PP. 38-85

31. Kenen P.J. «The Theory of Optimum Currency Areas: An Eclectic View», in Mundell R.A. and Swoboda A. K. (eds.), «Monetary Problems of the International Economy», University of Chicago Press, pp. 41-60, (1969).

32. Mantsie R. W. «inflation et croissance dans les pays de la CEMAC». Université Marien Ngouabi, Brazzaville- Congo, (2003).

33. McCallum B.T., «The case for rules in the conduct of monetary policy: a concrete example», Economic Review, Federal Reserve Bank of Richmond, (1987), sept.-oct., pp. 10-18.

34. McKinnon R. «Optimum Currency Areas», American Economic Review, $\mathrm{N}^{0} 53$, (1963), pp. 717-725.

35. Mosayed, P and Mohammad, R. «Sources of Inflation in Iran: An application of the real approach». International Journal of Applied Econometrics and Quantitative Studies, volume 6, issue1, (2009), pp.61 - 76.

36. Mundell, R. «A theory of Optimum Currency Areas», American Economic Review, September, vol. 51, (1961), pp. 657 - 665.

37. Nubukpo K. " L'impact de la variation des taux d'intérêt directeurs de la BCEAO sur l'économie béninoise». Note d'information statistique, $\mathbf{N}^{\mathbf{0}}$ 526, Banque des Etats de l'Afrique de l'Ouest, (2002).

38. Ouattara, B.; Strobl, E., , «Do Aid Inflows Cause Dutch Disease? A Case Study of the CFA Franc Countries», The School of Economics Discussion Paper, Series, (2003).

39. Ouattara, B.; Strobl, E., 2008) «Foreign Aid Inflows and the Real Exchange Rate in the Cfa Franc Zone», Economie Internationale, CEPII research center, issue 4Q, pp. 37-52.

40. Paulo, S. «L'Europe et la crise économique mondiale» Fondation Robert Shuman, (2011) Avril. 
41. Phillips A. W. « The Relationship between Unemployment and the Rate of Change of Money Wages in the United Kingdom, 18611957». Economica, 25. (1958),

42. Pichette, L. «La politique monétaire a-t-elle des effets asymétriques sur l'emploi?», Document de travail 98-17 de la Banque du Canada (1998), Septembre.

43. Rogoff, K. «The Optimal Degree of Commitment to an Intermediate Monetary Target », Quarterly Journal of Economics, 100, (1985), pp. 1169-1190,

44. Sarel, M. «Nonlinear effects of inflation on economic growth», Staff Papers. IMF Vol 43, No, 1; (1996), March.

45. Sfia, M. D. «Le choix du régime de change pour les économies émergentes», MPRA Working paper 4075. (2007).

46. Sahadudheen, I., «A cointegration and error correction approach to the determinants of inflation in India», International Journal of Economic Research, volume 3, issue 1, (2012), PP. 105-112.

47. Shari, S., «Politique Macroéconomique et politique de Croissance», Nations Unies Département des Affaires Economiques et Sociales, (2007), Juin.

48. Svensson L.E.O. «Open-Economy Inflation Targeting», Journal of International Economics, (2000), pp. 655-769.

49. Taylor, J.B. «Discretion versus Policy Rules in Practice», CarnegieRochester Conference Series on Public Policy, n 39, (1993).

50. Francis Kydland, Eric Prescott, " Rules rather than Discretion: The Inconsistency of Optimal Plans », Journal of Political Economy, 85, (1977), pp. 473-491. 


\section{ANNEXES: Tests de corrélation}

\section{Période entière}

- correlate tinfl ti tpibr m2 inv oil bc empl tch ce ( obs $=216$ )

\begin{tabular}{|c|c|c|c|c|c|c|c|}
\hline & tinfl & $\mathrm{ti}$ & tpibr & $\mathrm{m} 2$ & inv & oil & bc \\
\hline tinfl & $1 . \odot \odot \odot \odot$ & & & & & & \\
\hline $\mathrm{ti}$ & $-\odot . \odot \odot \odot 7$ & $1 . \odot \odot \odot \odot$ & & & & & \\
\hline tpibr & $-\odot .0695$ & $-\odot . \odot 331$ & $1 . \odot \odot \odot \odot$ & & & & \\
\hline $\mathrm{m} 2$ & $\odot .1756$ & $-\odot .0354$ & $\odot .126 \odot$ & $1 . \odot \odot \odot \odot$ & & & \\
\hline inv & $\odot .1807$ & $-\odot . \odot 625$ & $\odot .2878$ & $\odot .1642$ & $1 . \odot \odot \odot \odot$ & & \\
\hline oil & $-\odot . \odot 277$ & $-\odot . \odot \odot 63$ & $\odot .2538$ & ๑. 0191 & $-\odot .1544$ & 1. $\odot \odot \odot \odot$ & \\
\hline $\mathrm{bc}$ & $-\odot . \odot 298$ & $\odot .0126$ & $\odot .0376$ & $\odot .0682$ & $\odot .0343$ & $\odot .0213$ & $1 . \odot \odot \odot \odot$ \\
\hline empl & $-\odot . \odot \odot 74$ & $\odot .0227$ & $-\odot .1350$ & $\odot .4729$ & $-\odot . \odot 761$ & $-\odot .0445$ & $-\odot .0359$ \\
\hline $\mathrm{tch}$ & $\odot .5899$ & $\odot .0285$ & $-\odot .3846$ & $\odot .2242$ & $\odot .0121$ & $\odot .0504$ & $\odot . \odot 814$ \\
\hline \multirow[t]{2}{*}{$\mathrm{ce}$} & $-\odot .0366$ & $\odot .0210$ & -0.2455 & $\odot .3 \odot 94$ & $-\odot . \odot 2 \odot 1$ & $-\odot .0363$ & $-\odot .0148$ \\
\hline & empl & tch & ce & & & & \\
\hline empl & $1 . \odot \odot \odot \odot$ & & & & & & \\
\hline tch & $-\odot .0355$ & $1 . \odot \odot \odot \odot$ & & & & & \\
\hline $\mathrm{ce}$ & $\odot .2882$ & $\odot .0181$ & 1. $\odot \odot \odot \odot$ & & & & \\
\hline
\end{tabular}

\section{Première sous-période}

- correlate tinfl ti tpibr m2 inv oil bc empl tch ce ( obs=108)

\begin{tabular}{|c|c|c|c|c|c|c|c|}
\hline & tinfl & ti & tpibr & $\mathrm{m} 2$ & inv & oil & bc \\
\hline tinfl & 1. $\odot \odot \odot \odot$ & & & & & & \\
\hline ti & $\odot .0124$ & 1. $\odot \odot \odot \odot$ & & & & & \\
\hline tpibr & -0.1858 & $-\odot . \odot 473$ & $1 . \odot \odot \odot \odot$ & & & & \\
\hline $\mathrm{m} 2$ & $\odot .3498$ & $-\odot .1056$ & - ๑. . 0163 & 1. $\odot \odot \odot \odot$ & & & \\
\hline inv & $\odot .1737$ & $-\odot . \odot 838$ & $\odot .2188$ & $\odot .3212$ & $1 . \odot \odot \odot \odot$ & & \\
\hline oil & ๑. $\odot 9 \odot 3$ & $\odot . \odot \odot 18$ & $\odot .2151$ & 0.1070 & $-\odot .1945$ & $1 . \odot \odot \odot \odot$ & \\
\hline bc & $-\odot .0542$ & ๑. 0211 & $\odot .0511$ & $\odot .1358$ & $\odot .0776$ & $\odot .0126$ & $1 . \odot \odot \odot \odot$ \\
\hline empl & $\odot .0743$ & $\odot .0429$ & -0.1494 & $-\odot .0618$ & $-\odot .02 \odot 5$ & $-\odot .0109$ & $-\odot . \odot 258$ \\
\hline tch & $\odot .7 \odot 31$ & $\odot . \odot 479$ & $-\odot .4821$ & $\odot .5127$ & $\odot . \odot 86 \odot$ & $\odot . \odot 625$ & ๑. . 812 \\
\hline \multirow[t]{2}{*}{$\mathrm{ce}$} & 0.1311 & $\odot .0191$ & $\odot .0774$ & $\odot .2427$ & $\odot .0712$ & $-\odot . \odot 9 \odot 4$ & $\odot .0534$ \\
\hline & empl & $\mathrm{tch}$ & $\mathrm{ce}$ & & & & \\
\hline empl & 1. $\odot \odot \odot \odot$ & & & & & & \\
\hline $\mathrm{tch}$ & ๑. 0915 & $1 . \odot \odot \odot \odot$ & & & & & \\
\hline $\mathrm{ce}$ & $-\odot .3389$ & $\odot .1166$ & $1 . \odot \odot \odot \odot$ & & & & \\
\hline
\end{tabular}

\section{deuxième sous-période}

- correlate tinfl ti tpibr m2 inv oil bc empl tch ce ( obs=108)

\begin{tabular}{|c|c|c|c|c|c|c|c|}
\hline & tinfl & ti & tpibr & $\mathrm{m} 2$ & inv & oil & bc \\
\hline tinfl & $1 . \odot \odot \odot \odot$ & & & & & & \\
\hline ti & -0.1535 & 1. $\odot \odot \odot \odot$ & & & & & \\
\hline tpibr & $\odot .0985$ & -0.1238 & $1 . \odot \odot \odot \odot$ & & & & \\
\hline $\mathrm{m} 2$ & $-\odot .0624$ & $-\odot . \odot \odot 35$ & $\odot .1062$ & 1. $\odot \odot \odot \odot$ & & & \\
\hline inv & $\odot .2271$ & $-\odot . \odot 861$ & $\odot .3105$ & $-\odot .0311$ & $1 . \odot \odot \odot \odot$ & & \\
\hline oil & $-\odot .3122$ & $-\odot .0182$ & ๑. 3254 & $-\odot .0123$ & $-\odot .0969$ & $1 . \odot \odot \odot \odot$ & \\
\hline bc & $\odot .1317$ & $-\odot .0112$ & $\odot . \odot 853$ & $\odot .0584$ & -0.0363 & $\odot .0348$ & $1 . \odot \odot \odot \odot$ \\
\hline empl & $-\odot .1589$ & $-\odot .0994$ & -0.2699 & $\odot .6153$ & -0.2159 & $-\odot . \odot 394$ & -0.0123 \\
\hline tch & $-\odot .1093$ & $-\odot . \odot \odot \odot 3$ & $-\odot .2762$ & $\odot . \odot 4 \odot 7$ & $-\odot . \odot 989$ & $-\odot . \odot \odot 49$ & $-\odot .0132$ \\
\hline ce & $-\odot .1190$ & $\odot . \odot 485$ & $-\odot .3653$ & ๑. 3568 & -0.0588 & $-\odot . \odot 397$ & $-\odot .0249$ \\
\hline
\end{tabular}

\begin{tabular}{r|rrr} 
& empl & tch & ce \\
\hline empl & $1.0 \odot \odot \odot$ & & \\
tch & $-\odot . \odot 552$ & $1.0 \odot \odot \odot$ & \\
ce & 0.3135 & 0.1182 & $1.0 \odot \odot \odot$
\end{tabular}

